\title{
Simulación del Flujo en una Turbina Kaplan mediante Dinámica de Fluidos Computacional: Desarrollo de una Herramienta para Utilización Docente
}

\author{
José L. Vicéns, Blas Zamora y Antonio S. Kaiser \\ Universidad Politécnica de Cartagena, Depto. de Ingeniería Térmica y de Fluidos, \\ Doctor Fleming s/n, 30202 Cartagena-España (e-mail: agricol@msn.com, blas.zamora@upct.es, \\ antonio.kaiser@upct.es)
}

Recibido Jun. 17, 2011; Aceptado Ago. 04, 2011; Versión final recibida Ago. 18, 2011

\begin{abstract}
Resumen
Se presenta una propuesta de metodología docente para la asignatura "Energía Hidráulica y Energía del Mar" en el Máster de Energías Renovables de la Universidad Politécnica de Cartagena (España). A partir de la experiencia docente adquirida en dos cursos, se propone un enfoque didáctico acorde con el Plan Bolonia, para conseguir que el alumno adquiera competencias relacionadas con la capacidad de analizar y proyectar maquinaria hidráulica. Se ha elegido la simulación numérica del flujo a través de una turbina Kaplan. Se incluyen los enfoques de aprendizaje basado en problemas y basado en proyectos, y se incorpora además la figura del tutor facilitador como elemento clave. Se utiliza el software comercial Matlab como herramienta de traducción de geometría al programa mallador Gambit, desde donde se exporta la geometría, la malla y las condiciones de contorno al código CFD Ansys-Fluent. Se facilitará al alumno la adquisición de conocimientos sobre turbinas hidráulicas a través de distintos proyectos de simulación numérica.
\end{abstract}

Palabras clave: estudios de máster, máquinas hidráulicas, dinámica de fluidos computacional, aprendizaje con proyectos

\section{Flow Simulation in a Kaplan Turbine using Computational Fluid Dynamics: Development of a Teaching-Learning Method}

\begin{abstract}
This paper presents a teaching-learning methodology for the course "Hydropower and Ocean Power" in the Master of Renewable Energy at the Polytechnic University of Cartagena (Spain). From the experience gained in two courses, a teaching approach according to the Bologna Declaration, to ensure that students acquire skills related to the ability of analyzing and designing hydraulic machines is proposed. In particular, The numerical simulation of the flow through a Kaplan turbine was chosen for study. The method includes the problem-based learning and the project-based learning approaches, as well as the concept of facilitator-tutor as a key element of the method. The commercial software Matlab is employed as a tool to translate the geometry to the meshing software Gambit; from where the mesh and the boundary conditions are translated to the Ansys-Fluent CFD code. The aim is to provide the students with relevant knowledge on hydraulic turbines through different numerical simulation projects.
\end{abstract}

Keywords: master degrees, hydraulic machinery, computational fluid dynamics, project-based learning 


\section{INTRODUCCIÓN}

La docencia y la investigación en Energía Hidráulica y del Mar están relacionadas con dos aspectos destacables. El primero es la dificultad en la docencia de Mecánica de Fluidos, en donde la carencia de formación matemática juega un papel relevante. El segundo es el importante desarrollo de las áreas de conocimiento que atañen al control de los procesos. Ambos factores propician que en ocasiones la investigación en Energías Renovables deje a un lado el componente mecánico (y matemático) para centrarse en el aspecto de regulación, control y transporte de la potencia obtenida. El estudio del comportamiento del flujo en las turbinas hidráulicas (Radha Krishna, 1997) ocupa un lugar preponderante en distintas asignaturas de Ingeniería Industrial, Energética o Civil, por ejemplo. La formación básica de los alumnos de la "Energía Hidráulica y Energía del Mar", del Máster en Energías Renovables de la Universidad Politécnica de Cartagena (España), en cuanto al conocimiento de los parámetros fundamentales de una turbina hidráulica, se completa en esta asignatura con prácticas de diseño básico de sus partes principales. En Mecánica de Fluidos en general, la visualización y la comprensión del patrón de flujo es una parte fundamental que puede lograrse a través del uso de técnicas de simulación numérica CFD (Stern, 2004 y 2006; Çengel y Cimbala, 2006; Zamora y Kaiser, 2009; Zamora et al., 2010). En maquinaria hidráulica, puede destacarse como una de las principales dificultades en el proceso de enseñanza-aprendizaje la introducción de la geometría, lo que finalmente suele derivar este estudio a áreas relacionadas con la investigación y no con la docencia. Aunque puede encontrarse algún estudio interesante en la bibliografía (Pujol et al., 2010), lo cierto es que la mejora de la enseñanza en el área comentada ha recibido hasta el momento una atención limitada.

Se han impartido dos cursos académicos de la asignatura Energía Hidráulica y Energía del Mar. Con la experiencia adquirida, se ha emprendido un proceso de mejora del proceso de enseñanzaaprendizaje en la asignatura. Teniendo en cuenta que el perfil del alumno que cursa el Máster de Energías Renovables es en general diverso, se hace preciso establecer herramientas docentes capaces incluso de encauzar al alumno hacia tareas de investigación en Energías Renovables, independientemente de su camino curricular previo. La metodología que aquí se presenta se aplicará en el próximo curso, aunque ya ha empezado a ensayarse con alumnos que desarrollan su trabajo fin de máster en la materia relacionada con máquinas hidráulicas. La experiencia adquirida, en un momento en el que el sistema universitario español se está adaptando a las directrices del Espacio Europeo de Educación Superior (fijadas por el Ministerio de Educación y Ciencia de España, en el Real Decreto 1393/2007), puede servir como referencia general en la docencia de materias avanzadas que deban impartirse en el nuevo sistema de titulaciones.

En el presente trabajo, con un enfoque didáctico, se desarrolla un procedimiento simplificado para la simulación numérica del flujo de agua a través de una turbina hidráulica de tipo axial (Kaplan), utilizando el software comercial Matlab como herramienta de traducción de la geometría de la turbina al programa mallador Gambit, desde donde se exporta la geometría y el mallado al código CFD Ansys-Fluent, basado en un procedimiento de volúmenes finitos. Las decisiones de diseño se basan en la información obtenida en la bibliografía específica sobre el tema, y forman parte del proceso de aprendizaje. Se pretende pues aproximar al alumno de Ingeniería a la tecnología mecánica de las turbinas hidráulicas axiales, mediante una metodología docente acorde con las directrices generales establecidas en el Espacio Europeo de Educación Superior (EEES, o Plan Bolonia) (De Miguel et al., 2005); esto es, con una orientación hacia el aprendizaje del alumno para la adquisición de competencias concretas. Se aplicará el procedimiento desarrollado a partir de próximo curso académico como mejora del planteamiento pedagógico empleado. Además, la herramienta desarrollada puede aplicarse incluso en el ámbito de la investigación en máquinas hidráulicas, al permitir la discusión sobre la influencia de distintas aproximaciones en el mallado, o sobre los efectos de considerar distintos modelos de turbulencia, por ejemplo.

\section{METODOLOGÍA DOCENTE}

Esta propuesta implica la utilización de modalidades de enseñanza reconocidas para el desarrollo de competencias. Siguiendo el criterio aceptado para el crédito europeo ECTS (European Credit 
Transfer System), participan en este método las modalidades de clases teóricas y clases prácticas, talleres o seminarios, tutorías y trabajos guiados en grupo. En las clases teóricas, se utiliza el método expositivo, pero se confiere una especial importancia al aprendizaje basado en problemas (García et al., 2008; Araujo y Sastre, 2008) y al aprendizaje orientado a proyectos (POPBL) (Mills y Treagust, 2003-04), aplicándose el POPBL sobre todo en la parte más práctica de la asignatura, encaminada a adquirir la competencia de desarrollo de un proyecto profesional sobre energía hidráulica. Como herramientas de aprendizaje se propone emplear mapas conceptuales para detectar y corregir tanto obstáculos epistemológicos como carencias competenciales básicas o generales. Se utiliza el portafolios tanto para acotar las fuentes bibliográficas, así como para precisar los avances del alumno con vistas a su evaluación (Klenowsky, 2005), junto con la rúbrica. Se destacan a continuación dos elementos clave en el método: el tutor facilitador y la herramienta CFD.

En el desarrollo de esta propuesta la figura del tutor facilitador tiene un papel relevante, enfatizando su vertiente de "facilitador". Esta función facilitadora se inicia en la etapa de adquisición bibliográfica evitando una inversión excesiva en tiempo y trabajo por parte del alumno. Por un lado impide el desplazamiento del esfuerzo del alumno hacia el aprendizaje meramente operativo de los programas, en detrimento de la finalidad buscada, su aplicación, y por el otro lado, disuade del uso de estas mismas herramientas como cajas negras que no mejoran las competencias del alumno. Con estas actuaciones se pretende fomentar en el alumno las competencias metacognitivas constructivistas, que conducen a la actitud investigadora. Con este fin, el tutor facilitador ofrece desarrollos de los programas Matlab y Gambit detalladamente explicados para su lectura secuencial. En estos programas el alumno debe introducir los datos concretos por medio del teclado de ordenador en su ubicación adecuada. De este modo el estudiante recorre el programa apreciando las posibilidades operativas sin invertir en el aprendizaje de los propios programas un tiempo excesivo. De igual manera el tutor suministra subrutinas prediseñadas para hacer frente a las demandas de los alumnos.

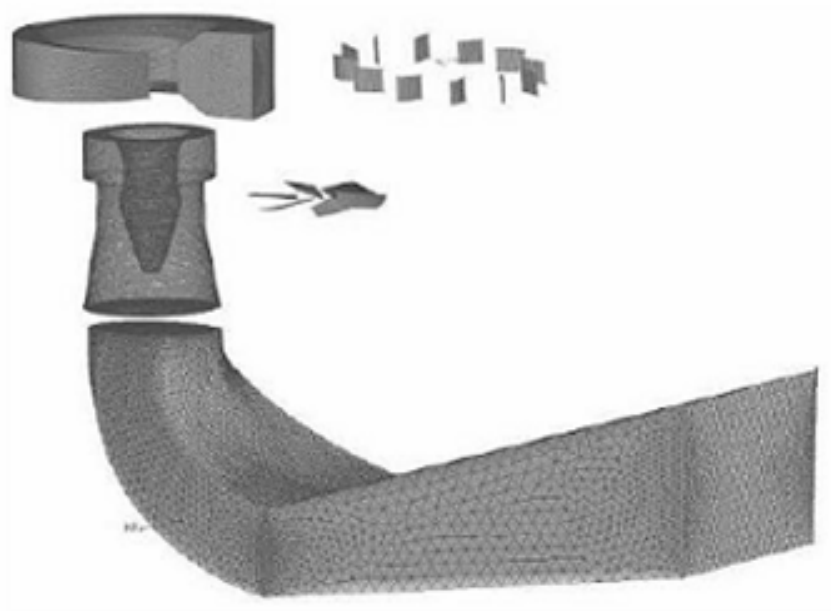

a)

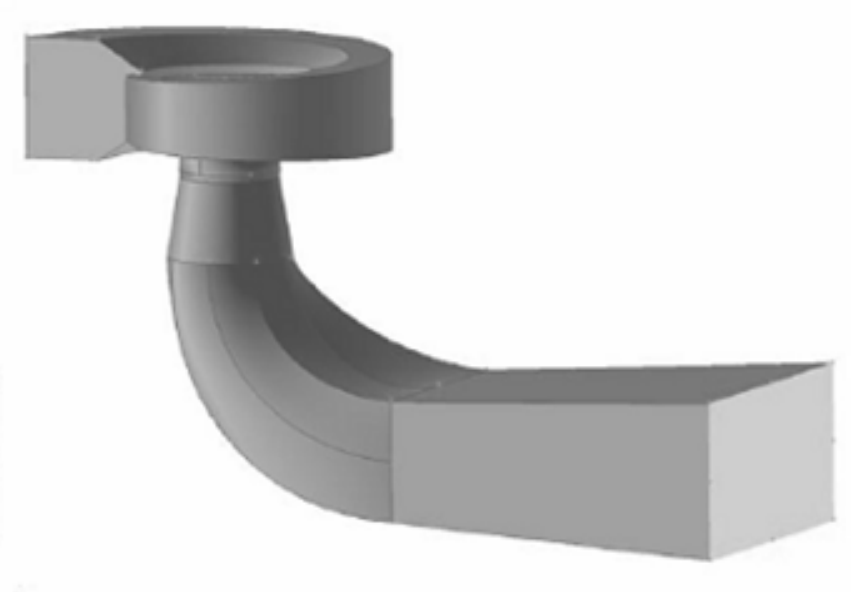

b)

Fig.1: Simulación numérica del flujo a través de una turbina Kaplan. a) Mallas de los componentes de la turbina que se diseñan y simulan aisladamente. b) Aspecto del modelo ensamblado.

La aplicación de la Dinámica de Fluidos Computacional (CFD) como herramienta docente (Navaz et al., 2002) es una parte esencial en nuestra propuesta. La CFD es una rama de la mecánica de fluidos que estudia los flujos resolviendo las ecuaciones de Navier-Stokes mediante métodos numéricos (Tu et al., 2008; Prasad et al., 2009). En Fluent, la simulación se lleva a cabo mediante el método de los volúmenes finitos; este código ha sido suficientemente validado y contrastado. En el preproceso se efectúa el diseño de la geometría y el mallado requerido (que en nuestro caso se lleva a cabo a través de Matlab y Gambit, respectivamente). En el proceso se efectúa la simulación (solución numérica de las ecuaciones con las simplificaciones adecuadas) con los 
parámetros escogidos: propiedades físicas, modelo de turbulencia, condiciones de contorno e iniciales, criterio de convergencia, o métodos de interpolación numérica, entre otros. El postproceso consiste en el análisis, interpretación y crítica de los resultados, y obviamente puede incluir un proceso retroactivo de estudio de sensibilidad de las simulaciones frente a los distintos factores.

\section{Escalones operativos docentes}

-Adquisición de elementos bibliográficos (tanto propuestos como libres), determinando los elementos de cálculo y los parámetros de simulación.

-Elaboración de programas en Matlab que proporcionan un croquis inicial, y las coordenadas que definen la geometría, a partir de los elementos de cálculo teóricos, y de los datos hidráulicos iniciales. Estos programas pueden contener también criterios de decisión.

-Exportación de las coordenadas a Gambit en forma matricial para construir la geometría y generar la malla. Los criterios de mallado a aplicar son los obtenidos en la búsqueda bibliográfica.

-Exportación de la malla a Fluent para proceder a la simulación CFD. Los parámetros de simulación se obtienen de la bibliografía y del tutor facilitador.

-Ensamblaje, si se desea, de las mallas de los distintos componentes de la turbina, para efectuar una simulación completa (véase la Fig. 1).

-Análisis de los datos obtenidos y estudio de la sensibilidad de la simulación con respecto a distintos parámetros.

\section{Elementos de apoyo de la tutoría}

a) Pool bibliográfico. Para permitir diversas propuestas de cálculo que puedan ser contrastadas ulteriormente se han seleccionado textos que ofrezcan opciones diversas. No obstante, esta bibliografía admite las aportaciones propias del alumno enriqueciendo "la oferta teórica". En lo referente a parámetros de simulación, la búsqueda se centra en "revistas de interés", disponiendo en la tutoría de una cuarentena de trabajos pertinentes publicados en los últimos años. Como en el caso de los elementos de cálculo, la bibliografía queda abierta a las aportaciones del alumno.

b) Cálculos con Matlab. Los programas de cálculo en esta aplicación se proporcionan al alumno según sus requerimientos de cálculo. Las rutinas más predecibles e inmediatas están disponibles de antemano. En todo caso, los programas vienen prolijamente documentados con líneas de comentarios. Se pretende que el alumno tome conciencia de las posibilidades que aporta esta herramienta de cálculo para la resolución de problemas, o el diseño y la investigación. El output de estos programas consta de matrices de coordenadas de puntos que definen las geometrías, croquis de estas geometrías y comprobaciones de idoneidad (Fig. 2).

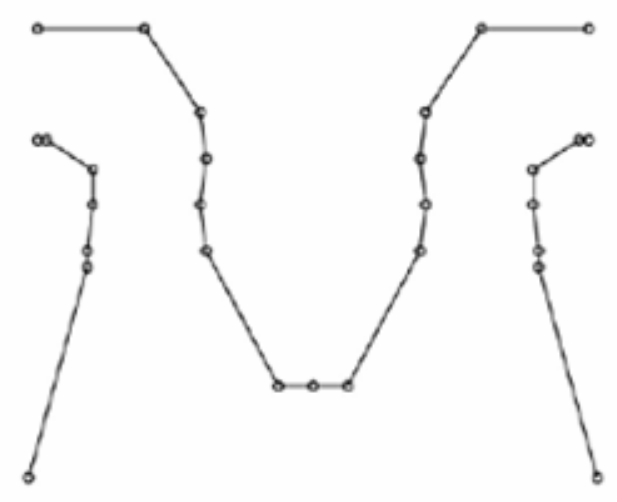

a)

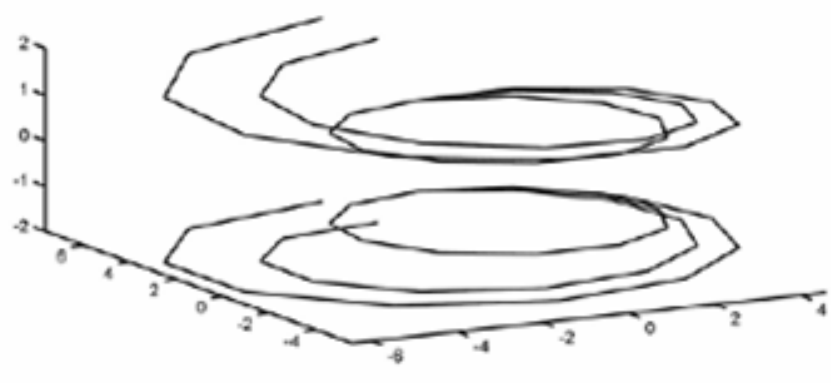

b)

Fig. 2: Ejemplos de croquis previos en el output de los programas Matlab. a) Croquis del canal del rotor en el que se muestran los puntos significativos a exportar. b) Croquis de la cámara espiral de sección t-shape generada en 3D. 
En las líneas siguientes se muestra un ejemplo de las comprobaciones de idoneidad que se van efectuando en los programas Matlab. En concreto, en el que proporciona los parámetros hidráulicos y geométricos del canal del rotor. Estas comprobaciones advierten de la idoneidad de la elección de la turbina Kaplan para la aplicación en cuestión, así como de la compatibilidad de los valores calculados con los requerimientos ingenieriles y con la información empírica disponible, para cada variante de turbina tipo Kaplan.

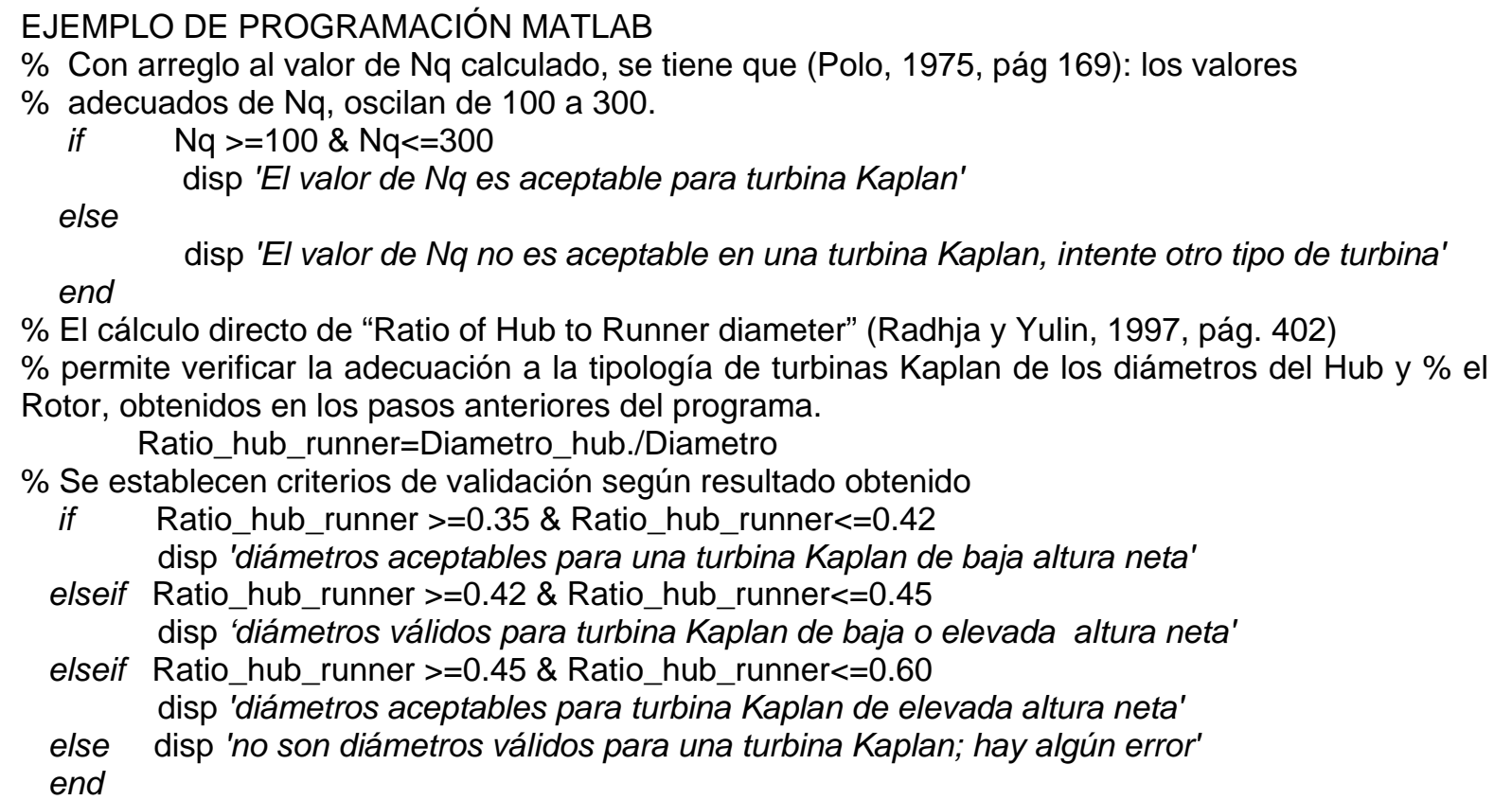

Puede comprobarse que se proporcionan al alumno, en las líneas del programa Matlab, las referencias bibliográficas en que se fundamentan los criterios de comprobación aplicados.

c) Geometría y mallado con Gambit. Como primer paso, el alumno debe efectuar un croquis a mano alzada y una triple proyección de los componentes asignados. Seguidamente, se procede a la construcción de la geometría a partir de las matrices de coordenadas proporcionadas por Matlab. El alumno tiene acceso a los ficheros de instrucciones de generación de malla tipo "journal" (".jou"), obtenidos en Gambit, y proporcionados por el tutor a la demanda del alumno según sus necesidades conceptuales de diseño. Los programas deben ser leídos secuencialmente por el alumno, que identifica su argumento y las operaciones que ejecuta. Igualmente modifica los parámetros pertinentes para ajustarlos al problema a resolver. Se pretende que el alumno perciba las potencialidades del programa, su versatilidad, y lo ejecute con sus propios parámetros, sin tener que invertir tiempo y recursos en su conocimiento específico, como se ha indicado anteriormente. Se dispone además de tutoriales propios, proporcionados por el tutor, que muestran de forma secuencial la construcción de las geometrías y su mallado. Como ejemplo, se listan a continuación unas líneas de un fichero “.jou”, correspondiente al diseño de la geometría de la corona de álabes del distribuidor.

\author{
EJEMPLO DE PROGRAMACIÓN GAMBIT \\ I Imponer la altura de los álabes del distribuidor \\ vertex cmove "vertex.24" multiple 1 offset 001.88 \\ edge create "altura vanes" straight "vertex.24" "vertex.33" \\ volume create "vane" translate "perfil vane" onedge "altura vanes" \\ I Introducir el ángulo del álabe con el radio del distribuidor \\ volume move "vane" dangle $\mathbf{4 5}$ vector 001 origin 000 \\ I Desplazar centrifugamente el álabe el radio del distribuidor \\ volume move "vane" offset $\mathbf{- 3 . 3 5} 00$ \\ I Generar los álabes para completar el distribuidor \\ volume cmove "vane" multiple 17 copyzone dangle 20 vector 001 origin 000
}


Puede observarse que el tipo de programación mostrada permite que el alumno inserte una determinada geometría (en este caso, un distribuidor tipo) sin que tenga que construir el programa ".jou". Al mismo tiempo, le permite configurar la geometría con arreglo a su intención de diseño, en este caso indicando el diámetro del distribuidor, así como el número y la altura de los álabes, y el ángulo de ataque de los mismos. El alumno debe modificar las cifras en negrita, introduciendo sus datos.

\section{RESULTADOS. PROYECTOS PROPUESTOS}

Para la realización de los proyectos propuestos, los alumnos disponen finalmente (elaborados por los alumnos y/o complementados por el tutor facilitador) de un conjunto de archivos Matlab, y Gambit (".jou"), perfectamente ajustables a distintas alternativas de diseño, correspondientes a la corona distribuidora, cámara espiral, álabe, álabe en túnel, hélice, hélice en conducción, rotor, tubo difusor, combinaciones de algunos de ellos y la turbina ensamblada en su totalidad. Si fuera necesario, el tutor puede facilitar los archivos de simulación Fluent (".cas" y ".dat") básicos para permitir que los alumnos puedan llevar a cabo una simulación inicial, a partir de la que se pueden desarrollar distintos proyectos. El postproceso puede orientarse tanto hacia la competencia específica sobre turbinas hidráulicas, como a las competencias trasversales de tratamiento de datos o de diseño de experimentos. A continuación, después de apuntar algunas notas sobre la simulación numérica, se exponen varios proyectos propuestos de forma muy resumida.

\section{Aspectos de la simulación numérica}

Se resuelven numéricamente las ecuaciones promediadas de Reynolds,

$$
\frac{\partial U_{j}}{\partial x_{j}}=0 ; \quad \frac{\partial\left(U_{i} U_{j}\right)}{\partial x_{j}}=-\frac{1}{\rho} \frac{\partial P}{\partial x_{i}}+\frac{\partial}{\partial x_{j}}\left(v \frac{\partial U_{i}}{\partial x_{j}}-\overline{u_{i} u_{j}}\right) \text {, }
$$

donde $U_{j}$ y $\mathrm{P}$ son la velocidad y la presión promediadas, respectivamente. El esfuerzo turbulento o aparente $-\overline{u_{i} u_{j}}$ se obtiene a través del modelo de turbulencia escogido, suponiendo que

$$
-\overline{u_{i} u_{j}}=2 v_{t} S_{i j}-\frac{2}{3} k \delta_{i j} ; \quad k=\frac{\overline{u_{x}^{2}}+\overline{u_{y}^{2}+\overline{u_{z}^{2}}}}{2}
$$

donde $v_{t}$ es la viscosidad cinemática turbulenta, $S_{i j}$ es el tensor de esfuerzos dado por $S_{i j}=$ $\left[\left(\partial U_{i} / \partial x_{j}\right)+\left(\partial U_{j} / \partial x_{i}\right)\right] / 2 ; \delta_{i j}$ la delta de Krönecker y $k$ la energía cinética turbulenta. Se han utilizado los modelos de dos ecuaciones de transporte $k-\varepsilon$ y $k-\omega$ (Wilcox, 2006). Aunque los mallados realizados conducen a obtener valores altos de la distancia adimensional desde el primer nodo de cálculo a la pared, $y^{+}$, se ha evaluado la influencia de considerar una mejora del $k-\omega$, el SST $k-\omega$, con buenos resultados. La turbulencia inicial se ha impuesto a través del concepto de intensidad de turbulencia, $I=[(2 / 3) k]^{1 / 2} / U$, con valores en torno al $10 \%$. Como se ha indicado, se utiliza un método de discretización de volúmenes finitos; se emplea además un procedimiento de interpolación "upwind" de segundo orden para los términos convectivos.

Proyecto 1. Diseño de los alabes del distribuidor. Análisis del flujo emergente frente a las variaciones del ángulo de ataque y del caudal del flujo incidente

El distribuidor actúa readaptando las componentes de la velocidad sobre el rotor. En clase, se explica cómo el distribuidor es el elemento clave de regulación del caudal y del intercambio de altura de Euler en el rodete. Estas modificaciones presentan una dificultad cognitiva para el alumno, de modo que su simulación y visualización ofrecen un evidente interés didáctico. Los resultados numéricos obtenidos se exportan a Matlab, para el tratamiento de los datos (Fig. 3). Se introduce al alumno en los procesos de representación sistemática de los datos, potenciando la idea de la superficie de respuesta como instrumento de comprensión de la influencia de las variables que intervienen en el problema (Fig. 3a). 


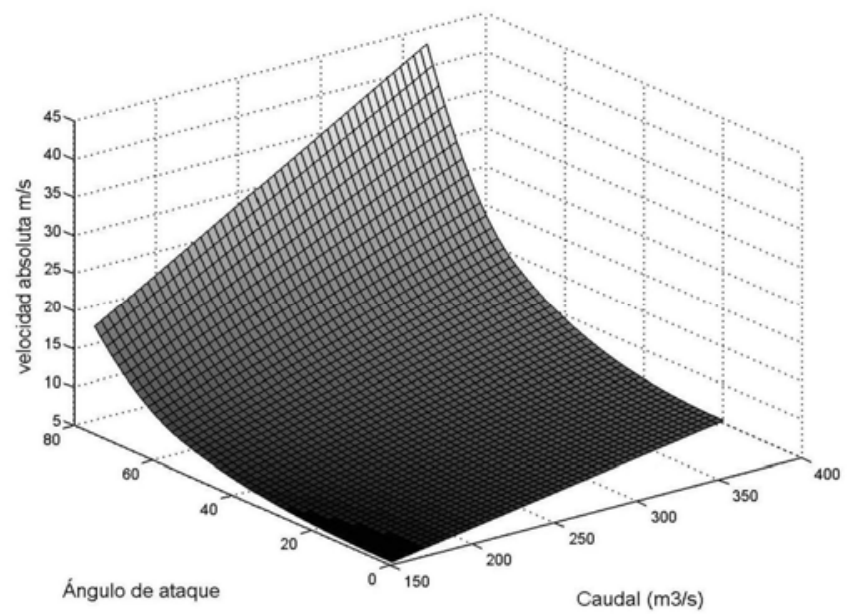

a)

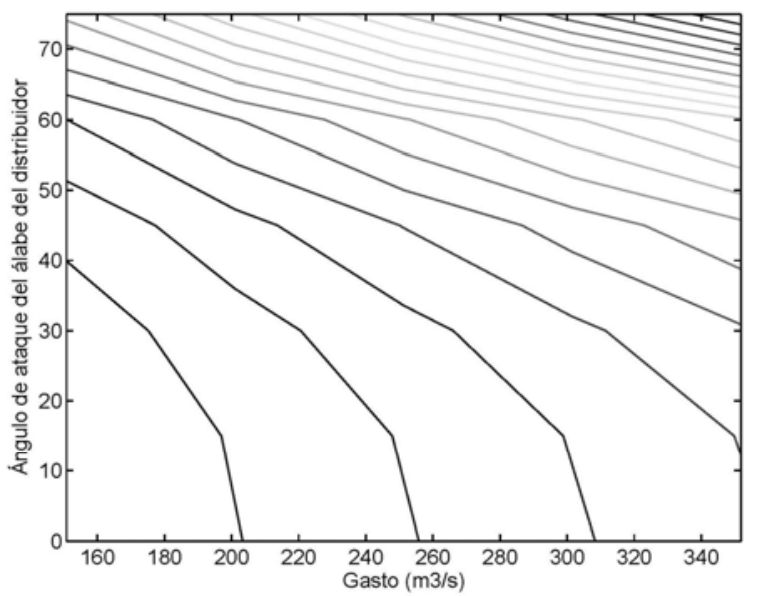

b)

Fig. 3: a) Superficie de respuesta de la velocidad absoluta de salida del distribuidor en función del ángulo de ataque y del caudal. b) Curvas de nivel (valor constante) de la velocidad absoluta del flujo eferente. Proyecto 1.

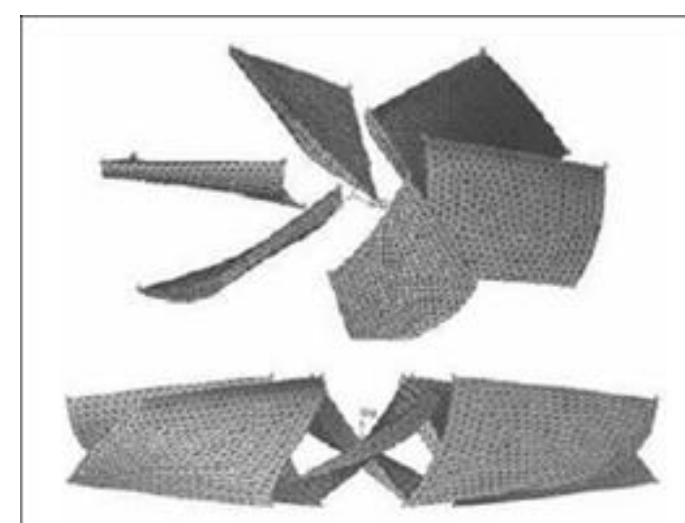

a)

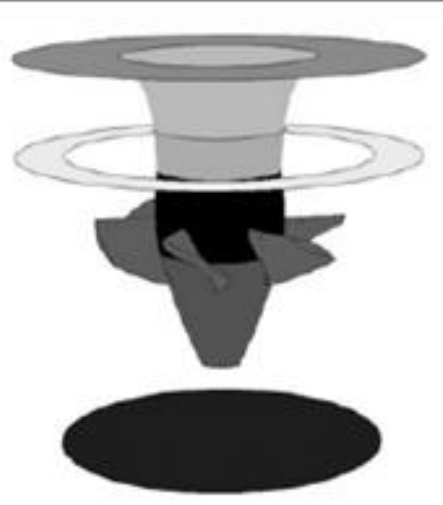

b)

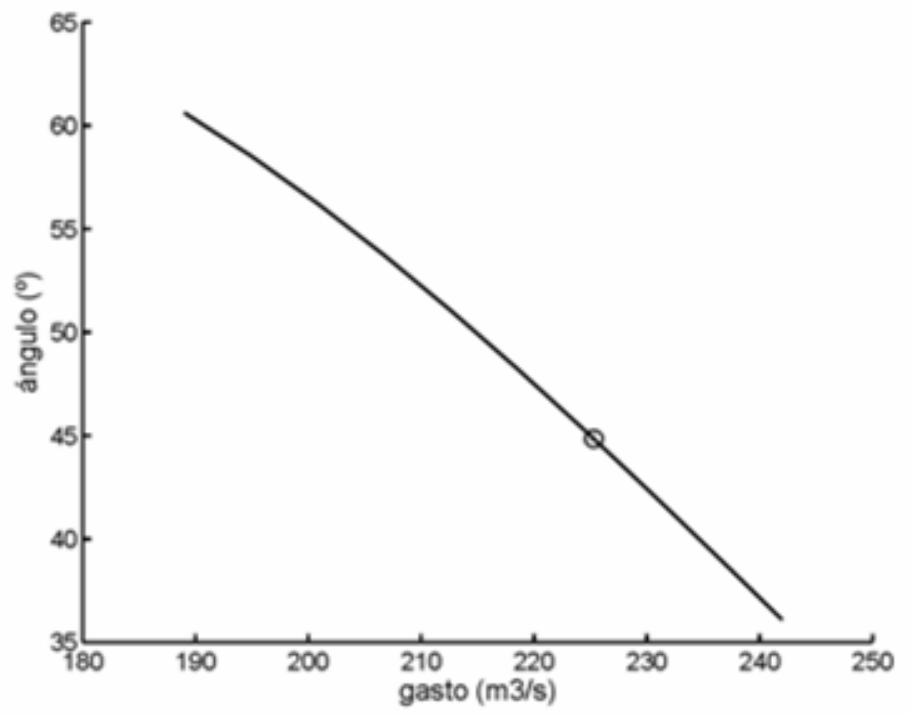

c)

Fig. 4: a) Mallado de los álabes. b) Despiece de algunos componentes aislables numéricamente. c) Curva de acoplamiento caudal-ángulo del distribuidor para mantener constante la potencia nominal obtenida (69 MW). Proyecto 2. 
Proyecto 2. Estudio de la potencia generada por la hélice en función del ángulo de ataque y del caudal del flujo incidente

Se realizan diversas simulaciones con un sistema rotor-hélice-canal del rotor, modificando el caudal y el ángulo de incidencia sobre los álabes para determinar la potencia absorbida por el rotor, determinada por la medida del momento cinético aplicado a la misma (Fig. 4).

Proyecto 3. Estudio de la eficiencia de la cámara espiral en función del caudal

La cámara espiral debe conducir de forma hidráulicamente eficiente al fluido hacia el rodete, de modo que es un elemento fundamental para obtener valores aceptables del rendimiento global del sistema. La Fig. 5 es un ejemplo de salida gráfica para llevar a cabo este proyecto. El grado de uniformidad de la distribución de presión en distintos planos puede ser una buena medida de la eficiencia de la cámara espiral.

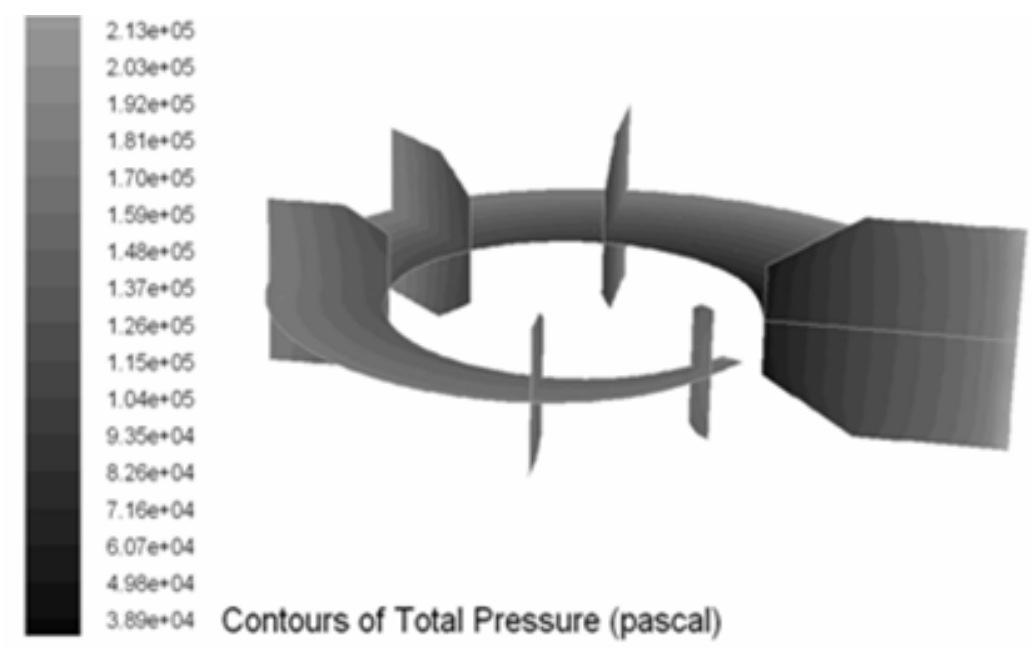

a)

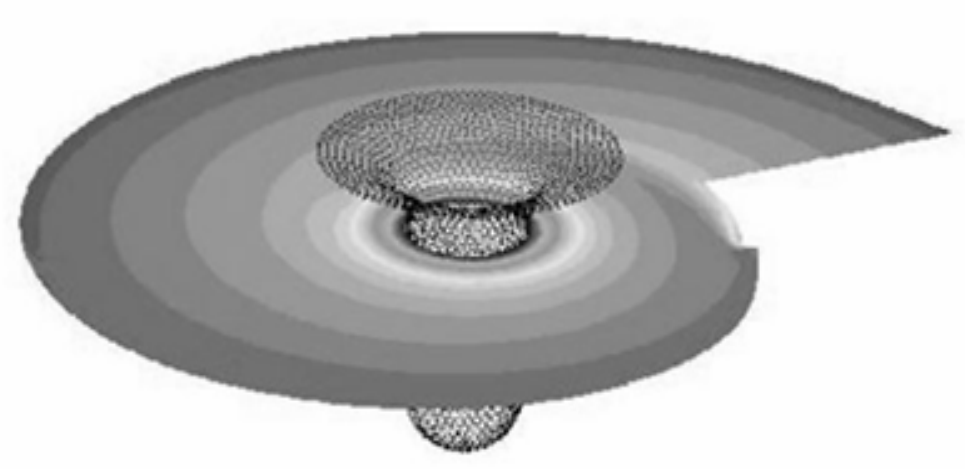

b)

Fig. 5: Visualización de la presión en diferentes planos definidos en la cámara espiral. a)

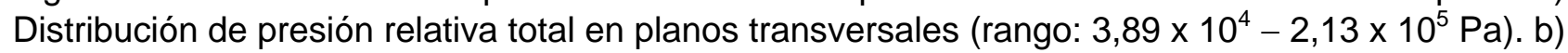
Distribución de la presión en un plano meridional, perpendicular al eje de rotación del rodete. Proyecto 3.

Proyecto 4. Detección e identificación de flujos secundarios. Determinación de los planos geométricos más significativos

En la Fig. 6 se muestra cómo puede analizarse el flujo tridimensional una vez ensamblados todos los componentes. Se observan los flujos secundarios en la salida del difusor. La complejidad de la 
geometría y la turbulencia del flujo restan coherencia a la suposición de considerar en la simulación un haz inicial de líneas de corriente en un pequeño sector del distribuidor, lo que indica que la axilsimetría empleada en algunas ocasiones en la bibliografía puede conducir a alguna simulación no realista. Este proyecto puede considerarse quizá como el más completo de los planteados, puesto que permite al alumno observar la totalidad del flujo en las distintas partes de la turbina, y vincular el patrón de flujo con la eficiencia global del sistema. La presencia de flujos secundarios y su influencia sobre el funcionamiento del sistema es un asunto complejo, que puede permitir al alumno emprender labores de investigación en el campo de las máquinas hidráulicas.

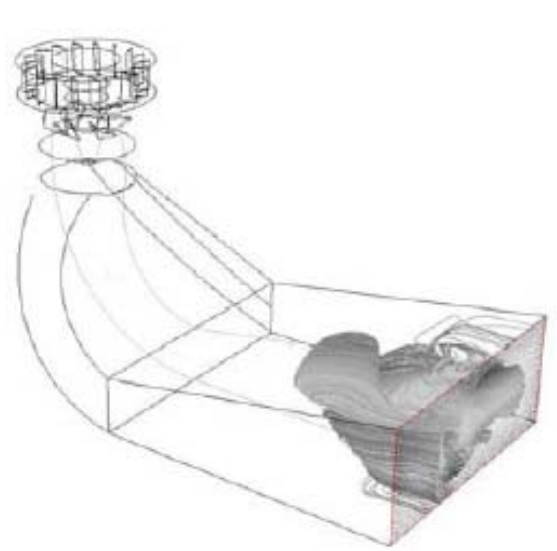

a)

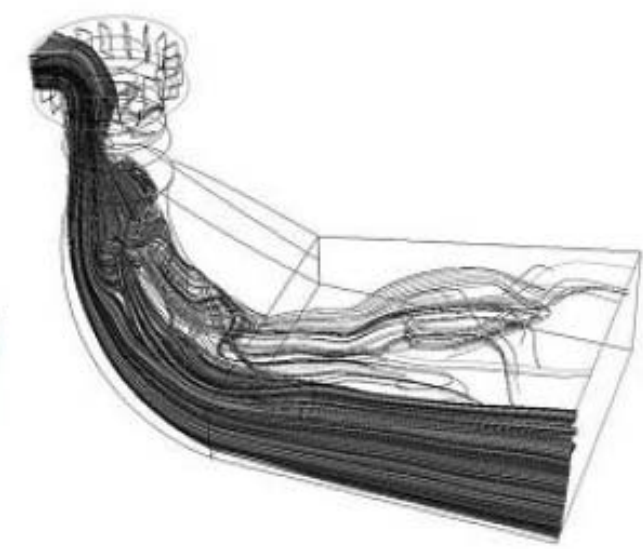

b)

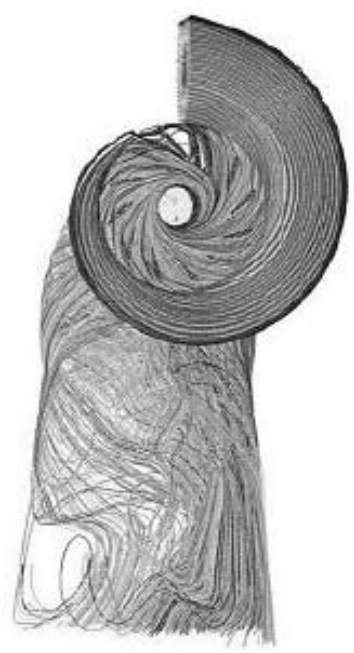

c)

Fig. 6: Caracterización del flujo sobre el sistema total ensamblado. Análisis de flujos secundarios utilizando las opciones de visualización del código CFD. Proyecto 4.

\section{CONCLUSIONES}

Puede concluirse que se ha desarrollado una herramienta que permite un acercamiento amistoso a un contenido árido que habitualmente ofrece problemas de rendimiento académico. Los autores creen que la herramienta desarrollada mejorará el nivel de la docencia impartida al superar los obstáculos epistemológicos a menudo detectados en el proceso de enseñanza-aprendizaje, tales como la concepción espacial bidimensional, la ausencia de visualización previa o el ansia de solución "analítica" de problemas geométricamente complejos. Puede remarcarse además que es una herramienta avanzada que es posible destinar a la investigación por la extensión del conocimiento concreto a un mayor detalle al presentarlo de forma más asequible, y por el progresivo aumento de las capacidades computacionales, que permiten estudiar la influencia de múltiples parámetros en el funcionamiento del sistema. Por último, es de prever la relevancia de la figura del tutor-facilitador en la consecución de los objetivos propuestos, por lo que es preciso prestar una especial importancia a la preparación y la predisposición de esta figura particular.

\section{REFERENCIAS}

Araujo, U.F. y G. Sastre; El Aprendizaje Basado en Problemas, Editorial Gedisa, Barcelona (2008).

Çengel, Y.A. y J.M. Cimbala, Mecánica de Fluidos (Fundamentos y Aplicaciones), Capítulo 15, McGraw-Hill, México (2006).

De Miguel, M., I.J. Alfaro, P. Apodaca, J. M. Arias, E. García, C. Lobato y A. Pérez; Metodologías de enseñanza y aprendizaje en el desarrollo de competencias. Orientaciones para el profesorado universitario ante el Espacio Europeo de Educación Superior. Alianza Editorial, Madrid (2006).

García, J., y otros veintiocho autores; El aprendizaje basado en problemas en la enseñanza universitaria, Servicio de Publicaciones de la Universidad de Murcia (2008). 
Klenowsky, V.; Desarrollo del portafolios para el aprendizaje y la evaluación, Nancea S.A. de Ediciones, Madrid (2005).

Navaz, H.K., B.S. Henderson, R.M. Berg y S.M.A. Nekcoei, A New Approach to Teaching Undergraduate Thermal/Fluid Sciences-Courses in Applied Computational Fluid Dynamics and Compressible Flow, The International Journal of Mechanical Engineering Education: 30(1), 35-49 (2002).

Mills, J., y D. Treagust, Engineering Education - Is Problem-Based or Project-Based Learning the Answer? Australasian Journal of Engineering Education, 2003-04, ISSN: 1324-5821 (en línea) http://www.aaee.com.au/journal/2003/mills_treagust03.pdf. Acceso: 16 de Agosto (2011).

Ministerio de Educación y Ciencia de España [en línea], Real Decreto 1393/2007, por el que se establece la ordenación de las enseñanzas universitarias oficiales, BOE $\mathrm{n}^{\circ} 270$ de 30 de Octubre de 2007, pp. 44037 a 44048. http://www.boe.es/boe/dias/2007/10/30/pdfs/A44037-44048.pdf. Acceso: 16 de Agosto (2011).

Prasad, V., Gahlot, V.K., Krishnamachar, P.; CFD Approach for Design Optimization and Validation for Axial Flow Hydraulic Turbine, Indian J. Engineering \& Materials Sciences: 13, 229236 (2009).

Pujol, T., Montoro, L., Pelegrí, M., González, R., Learning Hydraulic Turbomachinery with Computacional Fluid Dynamics (CFD) Codes, Computers Applications in Engineering Education, DOI: 10.1002/cae.20513 (2010).

Radha Krishna, H.C.; Hydraulic Design of Hydraulic Machinery, Avebury (1997).

Stern, F., y otros diez autores, Development of Hands-On CFD Educational Interface for Undergraduate Engineering Courses and Laboratories, ASEE Annual Conference Proceeding, 3719-3749, Salt Lake City, USA (2004).

Stern, F., y otros diez autores, Hands-On CFD Educational Interface for Engineering Courses and Laboratories, Journal of Engineering Education: 95(1), 63-83 (2006).

Tu, J., Yeoh, G.H., Liu, C.; Computational Fluid Dynamics: A Practical Approach, Elsevier (2008).

Wilcox, D.C., Turbulence Modeling for CFD, 3a ed., DCW Industries (2006).

Zamora, B., Kaiser, A.S., Enseñanza de temas avanzados de Mecánica de Fluidos usando Dinámica de Fluidos Computacional, Formación Universitaria: 2(1), 27-39 (2009).

Zamora, B., Kaiser, A.S., Vicente, P.G., Improvement in Learning on Fluid Mechanics and Heat Transfer Courses Using Computational Fluid Dynamics, International Journal of Mechanical Engineering Education: 38(2), 147-166 (2010). 